\title{
Estudio comparativo del reconocimiento de rostros térmicos basado en características invariantes
}

\author{
Raúl Aguilar Figueroa ${ }^{1}$, Raúl Santiago Montero ${ }^{1}$, \\ Juan Humberto Sossa Azuela ${ }^{2,3}$ \\ ${ }^{1}$ Instituto Tecnológico de León, \\ División de Estudios de Posgrado e Investigación, Guanajuato, \\ México \\ ${ }^{2}$ Instituto Politécnico Nacional, Centro de Investigación en Computación, \\ México \\ 3 Tecnológico de Monterrey, Campus Guadalajara, Zapopan, Jalisco, \\ México \\ $\{$ rauly.af123, rsantiago66\}@gmail.com, \\ hsossa@cic.ipn.mx
}

Resumen. Históricamente, el reconocimiento automático de rostros se ha enfocado en el espectro visible. Sin embargo, este enfoque se ve afectado por una serie de factores que se atribuyen, principalmente, a la variación en la iluminación, a la dificultad para detectar disfraces faciales y a los cambios en las poses y expresiones faciales. Para superar estos inconvenientes, surge la alternativa de las imágenes térmicas. No obstante, estas imágenes no están exentas de limitantes, tales como: cambios en la temperatura ambiente, variaciones en el metabolismo y la recolección de datos de prueba en diferentes lapsos de tiempo. Entre los diversos métodos que se han desarrollado para obtener representaciones térmicas del rostro más estables, sobresalen dos: la extracción de la red de vasos sanguíneos y la extracción de la perfusión sanguínea. En el presente artículo, se proponen dos metodologías de reconocimiento de rostros térmicos: la primera combina los métodos de extracción de la red de vasos sanguíneos y la perfusión sanguínea; mientras que la segunda metodología hace uso exclusivo de la extracción de la red de vasos sanguíneos. En ambas metodologías, se utiliza el descriptor Factor- $E$ Normalizado (FEN) para la extracción de características, mientras que la clasificación se realiza por medio de una máquina de soporte vectorial y un bosque aleatorio. Los resultados experimentales demuestran que el reconocimiento de rostros de la segunda metodología, alcanza una tasa de reconocimiento comparable al de la primera metodología.

Palabras clave: reconocimiento facial, imagen térmica, red de vasos sanguíneos, perfusión sanguínea. 


\title{
A Comparative Study of Thermal Face Recognition Based on Invariant Characteristics
}

\begin{abstract}
Historically, automatic face recognition has focused on the visible spectrum. However, this approach is affected by a series of factors that are attributed, mainly, to variation in lighting, to the difficulty in detecting facial costumes and the changes in poses and facial expressions. To overcome these drawbacks, thermal images arise as alternative. Nevertheless, these images are not exempt from limitations such as: changes in ambient temperature, variations in metabolism and time-lapse testing. Among various methods that have been developed to obtain more stable thermal representations of face, two stand out: the extraction of the blood vessel network and the extraction of the blood perfusion. In this paper, two methodologies are proposed for thermal face recognition: the first combines the methods of extraction of the blood vessel network and blood perfusion; while the second methodology makes exclusive use of the extraction of the blood vessel network. In both methodologies, the Normalized E-Factor is used for the extraction of characteristics, while classification is carried out by means of a vector support machine and a random forest. The experimental results show that the face recognition of the second methodology, reaches a recognition rate comparable to that of the first methodology.
\end{abstract}

Keywords: face recognition, thermal image, blood vessel network, blood perfusion.

\section{Introducción}

El reconocimiento automático de rostros se ha constituido como una disciplina de especial interés para los sectores académico y comercial, debido a la amplia variedad de aplicaciones que presenta, entre las que se encuentran: sistemas de vigilancia, control de acceso a instalaciones seguras y sistemas de entretenimiento $[10,12,15]$. Históricamente, los métodos desarrollados para el reconocimiento automático de rostros, se han enfocado en el uso de imágenes captadas en el espectro visible [5]; sin embargo, estas imágenes se ven afectadas por una serie de factores que disminuyen la precisión en el reconocimiento, y que se atribuyen, principalmente, a la variación en la iluminación, a la dificultad para detectar disfraces faciales y a los cambios en las poses y expresiones faciales $[12,16,29]$.

Buscando solucionar los problemas presentes en los sistemas de reconocimiento de rostros basados en el espectro visible, surge la alternativa de las imágenes captadas en el espectro infrarrojo, conocidas como imágenes infrarrojas $[9,24,29,32]$. El espectro infrarrojo se divide en cuatro bandas: infrarrojo cercano $(0.7-0.9 \mu \mathrm{m})$, infrarrojo de onda corta $(0.9-2.4 \mu \mathrm{m})$, el infrarrojo medio $(3.0-5.0 \mu \mathrm{m})$ y el infrarrojo lejano $(8.0-14.0 \mu \mathrm{m})$ [11]. La mayor cantidad 
de emisión de energía térmica por parte del cuerpo humano se produce en la banda del infrarrojo medio y en la del infrarrojo lejano; es por esta razón que ambas bandas del espectro infrarrojo, que conforman la llamada banda térmica infrarroja, han recibido la mayor atención en la literatura del reconocimiento automático de rostros [10,13]. La representación térmica facial está determinada por la red vascular subcutánea del rostro, la cual es irreproducible y por lo tanto, única para cada persona [17]. Esto representa una posibilidad para realizar una extracción no invasiva de información anatómica característica de cada persona, que puede ser usada para el reconocimiento de rostros [8].

Entre las ventajas que ofrecen las imágenes captadas en el espectro térmico infrarrojo (también llamadas termogramas), con respecto al espectro visual, se encuentran las siguientes: independencia ante la iluminación externa, detección de disfraces faciales, y una mayor robustez frente a las variaciones en las poses y expresiones faciales $[11,12,16,22]$. No obstante, los termogramas no están exentos de limitantes, pues son afectados por una serie de factores tales como: cambios en la temperatura ambiente, variaciones en el metabolismo, práctica de ejercicio, patrones de respiración y la recolección de datos de prueba en diferentes lapsos de tiempo $[5,6,25,27,30,31]$. Además, es importante señalar que otro de los problemas presentes en los termogramas se debe a la opacidad de los anteojos ante la banda infrarroja térmica, lo que resulta en pérdida de información cercana a los ojos $[5,16,25,30,31]$.

Con el objetivo de obtener una representación térmica invariante de cada rostro, y así superar las limitantes de los sistemas de reconocimiento de rostros térmicos, las investigaciones se han encaminado en dos vertientes: la extracción de la red de vasos sanguíneos del rostro y la extracción de la perfusión sanguínea del rostro $[1,4,22]$. En el presente artículo, se realiza un estudio que combina los dos métodos de extracción de características térmicas antes mencionados, tomando como base las propuestas que dieron origen a dichos métodos: para el caso de la red de vasos sanguíneos, la referencia son los artículos publicados por Buddharaju et al. [3-5], mientras que la propuesta para obtener la perfusión sanguínea del rostro, se encuentra en los trabajos de Wu et al. [28,29]. En nuestro análisis, se establecen dos metodologías. En la primera, el enfoque de Buddharaju et al. se implementa sobre la propuesta de $\mathrm{Wu}$ et al. Posteriormente, haciendo uso del descriptor Factor- $E$ Normalizado (FEN) [19], se define un vector de características de nueve dimensiones. Finalmente, el proceso de clasificación se realiza por medio de dos métodos distintos: una máquina de vector soporte y un bosque aleatorio. El análisis propuesto se implementó en los termogramas de la base de datos Terravic Facial $\mathrm{IR}^{1}$, los cuales presentan variaciones en las poses del rostro (frente, izquierda, derecha) y en el entorno (interior, exterior), además de contener accesorios como lentes y gorras. La segunda metodología consiste en implementar únicamente el enfoque de Buddharaju et al., mientras que el resto de las etapas del sistema de reconocimiento, son las mismas que las especificadas

${ }^{1}$ IEEE OTCBVS WS Series Bench; Roland Miezianko, Terravic Research Infrared Database. 
para la primera metodología. Al final, se realiza una comparación entre el rendimiento alcanzado por ambas metodologías durante el reconocimiento de rostros.

El resto del artículo se organiza de la siguiente manera. En la Sección 2, se describen los enfoques de Buddharaju et al. y Wu et al., antecedidos por un resumen de los primeros trabajos que buscaban extraer características de las imágenes infrarrojas. El descriptor FEN y los algoritmos de clasificación empleados para la tarea de reconocimiento, son analizados en la sección 3. Los detalles de las metodologías propuestas, se delinean en la Sección 4. En la Sección 5 se presentan los resultados experimentales y un análisis de los mismos. Las conclusiones y el trabajo futuro se establecen en la Sección 6 .

\section{Antecedentes}

\subsection{Propuestas iniciales referentes a la extracción de características del rostro térmico}

La extracción de características únicas para cada individuo a partir de los termogramas, fue sugerida por Prokoski et al. [18], sin embargo, su estudio se limitó al aspecto teórico y no presentaron una implementación de un sistema de reconocimiento de rostros.

Uno de los esfuerzos iniciales por desarrollar un método de reconocimiento de rostros basado en la extracción de características faciales de los termogramas, proviene del trabajo de Yoshitomi et al. [23]. En esta propuesta, se realizó la extracción de los niveles de gris de los termogramas y se emplearon como un primer descriptor. Así mismo, fueron utilizados como descriptores los valores obtenidos por los factores de forma 1 y 2 . A pesar de que la tasa de reconocimiento de rostros térmicos en condiciones internas y externas alcanzó valores notables $(100 \%$ y $97.5 \%$, respectivamente), los termogramas usados corresponden exclusivamente a rostros captados en posición frontal, y en condiciones controladas; de tal forma que las variaciones en las poses y expresiones faciales, así como la captura de imágenes en situaciones no controladas, no fueron tomadas en cuenta en este estudio y por lo tanto, se desconocen los efectos del método propuesto ante un escenario dinámico.

Li et al. [13-16], presentaron un mecanismo para la extracción de patrones binarios locales de las imágenes infrarrojas. Como parte de su metodología, emplearon imágenes del infrarrojo cercano para establecer un sistema de reconocimiento de rostros. El proceso de reconocimiento de rostros se realizó únicamente en condiciones internas y como era de esperarse, los resultados que se obtuvieron arrojaron una alta precisión. Es importante señalar que para la captura de las imágenes en el infrarrojo cercano, es necesaria la cooperación del usuario, lo que representa un escenario opuesto a la mayoría de las situaciones prácticas en que se captan las imágenes infrarrojas y además, debido a que luz solar tiene una elevada composición espectral en la longitud de onda del infrarrojo cercano, los sistemas que utilizan este tipo de imágenes, son deficientes en el reconocimiento de rostros en ambientes externos [8]. 


\subsection{Método para la extracción de la red de vasos sanguíneos del rostro térmico}

Fueron los trabajos de Buddharaju et al. [3-5] los que definieron un nuevo paradigma para la extracción de características únicas e invariantes del rostro térmico. Esta propuesta se sustenta en la compleja combinación de venas y arterias faciales que conforma un patrón característico para cada individuo.

La metodología para el reconocimiento de rostros térmicos propuesta por Buddharaju et al., consta de los siguientes puntos:

1. Segmentación del rostro con respecto al fondo de la imágen térmica.

2. Reducción del ruido y resaltado de los bordes del rostro segmentado mediante un filtro de difusión anisotrópica.

3. Extracción de la red de vasos sanguíneos del tejido facial a través de la transformada top-hat blanca.

4. Adelgazamiento de la red vascular extraída y extracción de los puntos de ramificación de la red vascular, denominados Puntos Característicos Térmicos (TMPs por sus siglas en inglés).

5. Los TMPs extraídos del rostro del individuo en cuestión, se almacenan en la base de datos.

6. La etapa de prueba consiste en extraer las estructuras locales y globales de los TMPs de la imagen térmica de prueba y compararlas con los TMPs almacenados en la base de datos.

En la Figura 1 Las características de esta base de datos pueden consultarse en la Sección 5 del presente artículo.

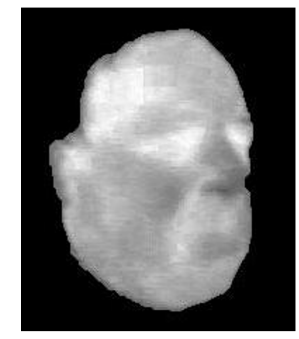

(a)

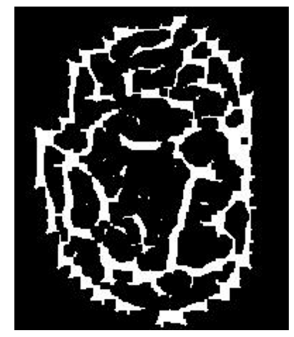

(b)

Fig. 1. Extracción de la red de vasos sanguíneos. (a) Imagen térmica segmentada (b) Vasos sanguíneos extraídos usando la transformada top-hat blanca.

\subsection{Método para la extracción de la perfusión sanguínea del rostro térmico}

Wu. et al. [22] propusieron un enfoque que también utiliza características fisiológicas invariantes para el reconocimiento de rostros. Dicha propuesta describe 
un modelo matemático basado en la termodinámica y en la fisiología térmica, y que convierte los termogramas en información de perfusión sanguínea. En su propuesta, $\mathrm{Wu}$ et al. realizaron la extracción de características fisiológicas a través del método de Análisis de Componentes Principales y el Discriminante Lineal de Fisher y para el proceso de clasificación, hicieron uso de una red neuronal de función de base radial.

En un esfuerzo posterior, Wu et al. [21] modificaron su propuesta original y definieron un modelo más simple y fácil de comprender. Los resultados mostraron que el desempeño de ambos modelos era comparable y superaba al enfoque basado en información térmica cuando son sometidos a los efectos de la variación en la temperatura del medio ambiente, cambios en el metabolismo, uso de anteojos, variaciones en los patrones de respiración y pruebas realizadas en lapsos de tiempo distintos. De hecho, el modelo de perfusión sanguínea modificado es superior al modelo original cuando son comparados en términos de experimentos de prueba realizados en lapsos de tiempo distintos. El modelo de perfusión sanguínea es una transformación no lineal que se aplica a cada píxel de la imagen. La ecuación (1) expresa la definición matemática del modelo de perfusión sanguínea modificado:

$$
W=\frac{\epsilon \sigma\left(T^{4}-T_{e}^{4}\right)}{\alpha c_{b}\left(T_{a}-T\right)},
$$

donde $\epsilon=0.98$ es la emisividad de la piel, $\sigma=5.67 * 10^{-8} \mathrm{~W} /\left(\mathrm{m}^{2} \mathrm{~K}^{4}\right)$ es la constante de Stefan-Boltzmann, $\alpha=0.8$ es la razón de intercambio a contracorriente, $c_{b}=3.78 * 10^{3} \mathrm{~J} /(\mathrm{kgK})$ es el calor específico de la sangre, $T_{a}=312.15 \mathrm{~K}$ es la temperatura interna del cuerpo, $T_{e}$ es la temperatura del medio ambiente, y $T$ es la temperatura de la superficie de la piel.

En el presente artículo, se hace uso del modelo modificado de Wu et al. para la extracción de la perfusión sanguínea del rostro. La implementación de dicho modelo en una de las muestras de la base de datos Terravic Facial IR Database, se observa en la Figura 2.

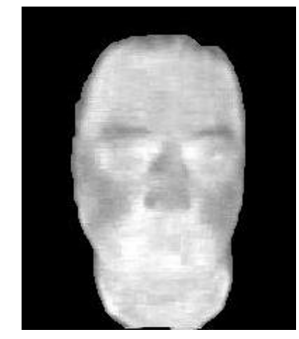

(a)

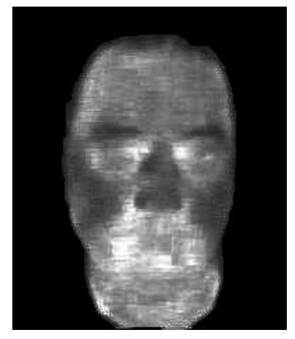

(b)

Fig. 2. Extracción de la perfusión sanguínea del rostro (a) Imagen segmentada original (b) Imagen de perfusión sanguínea. 
Hasta ahora, el primer y único esfuerzo por comparar el rendimiento de las propuestas de Buddharaju et al. y Wu et al., se encuentra en el trabajo de Konuk [12]. En dicho trabajo, se realizó por primera vez la combinación de ambas propuestas y la comparación entre las mismas. Al combinar las propuestas, la metodología empleada para la clasificación de termogramas, es la misma que la establecida por Buddharaju et al., con la diferencia de que la extracción de los TMPs se lleva a cabo a través de otros métodos de extracción. El proceso de clasificación fue implementado en dos bases de datos, y en ambas, la mejor tasa de reconocimiento fue alcanzada tanto por el enfoque que emplea únicamente la red de vasos sanguíneos como por el que combina la perfusión sanguínea con la red de vasos sanguíneos. Las tasas de reconocimiento para las dos bases de datos fueron de $99.5 \%$ y $98.1 \%$. A pesar de estos resultados, es importante remarcar que para la etapa de prueba, en las dos bases de datos se utilizaron muy pocos termogramas y además, el autor no especifica el mecanismo para la elección de estos termogramas de prueba y por lo tanto, no es posible establecer una conclusión general acerca del comportamiento de los métodos propuestos.

\section{Descriptor y algoritmos de clasificación}

\subsection{Descriptor Factor- $E$ Normalizado (FEN)}

El Factor- $E$ Normalizado es un descriptor de forma basado en región, que se define como una medida de compacidad simple, robusta a traslaciones, rotaciones, transformaciones de escala y libre de inconsistencias en su diseño [19].

$\mathrm{Al}$ ser un descriptor basado en regiones, aprovecha la información de los bordes y del interior de la forma para generar descriptores de forma, y además, es posible generar la descripción de la forma a partir de la división de la misma en partes más pequeñas [24].

La ecuación (2) determina la expresión matemática del FEN en el espacio digital 2D:

$$
F E N=\frac{P_{\text {shape }}}{4 \sqrt{n}}
$$

donde $P_{\text {shape }}$ corresponde al perímetro de la forma en cuestión y $4 \sqrt{n}$ corresponde al perímetro de un cuadrado con el mismo número de elementos que la forma original.

\subsection{Máquina de vector soporte}

Las máquinas de vector soporte (SVM, del inglés Support Vector Machine) son algoritmos supervisados que realizan tareas de clasificación y regresión [20]. El objetivo de las SVMs es la generación de fronteras de decisión a partir del conjunto de entrenamiento para clasificar instancias que pertenezcan a dos clases distintas [7].

La frontera de decisión se encuentra al mapear los datos de entrenamiento hacia un espacio de mayores dimensiones (este espacio puede llegar a ser infinito), 
donde la frontera de decisión se representa por un hiperplano que utiliza un margen para alcanzar la mayor separación posible entre las instancias correspondientes a las dos clases $[6,10]$. Con base en lo anterior, se dice que una SVM es un estimador de margen máximo [20].

La proyección de los datos hacia un espacio de mayores dimensiones, es un proceso computacional muy costoso $[6,20]$. Para resolver este inconveniente, se hace uso de la función kernel, que es un mecanismo que evita calcular las coordendas de los datos en el nuevo espacio dimensional, y procede a determinar únicamente la distancia entre pares de puntos en dicho espacio [6].

\subsection{Bosques aleatorios}

Los bosques aleatorios son algoritmos de clasificación no paramétricos. Se caracterizan por ser métodos combinados, los cuales, durante el proceso de clasificación, consideran los resultados de un conjunto de estimadores simples para determinar la precisión en la predicción [20].

Los árboles de decisión son los estimadores simples sobre los que se construyen los bosques aleatorios [2]. Cada árbol de decisión se define por un vector aleatorio probado independientemente. Una votación mayoritaria se establece entre los árboles de decisión que conforman el bosque aleatorio, determinando de esta forma la clase más popular. A través del aumento de los árboles de decisión combinados, se alcanza una mayor precisión en la clasificación.

\section{Metodologías para el reconocimiento de rostros térmicos}

En esta Sección, se describen las dos metodologías propuestas en este artículo para la tarea de reconocimiento de rostros térmicos. En la primera metodología, el método de extracción de vasos sanguíneos propuesto por Buddharaju et al. se aplica sobre el modelo de perfusión sanguínea propuesto por Wu et al. La segunda metodología consiste en implementar únicamente el método de Buddharaju et al. sobre los termogramas.

Debido al hecho de que las metodologías propuestas difieren sólo en la representación térmica, la descripcion de las metodologías se unifica, haciendo énfasis en el punto en que ambas difieren.

\subsection{Segmentación del rostro}

El proceso de segmentación del rostro se basa en el procedimiento encontrado en [9] y consiste en la creación de una máscara de segmentación provisional, en la que todos los píxeles que se encuentran en un rango definido por dos umbrales, toman el valor de 1 y se definen como parte del rostro, mientras que los píxeles restantes toman el valor de 0 y se consideran como parte del fondo.

Posteriormente, el mapa de segmentación provisional se refina por medio de las operaciones morfológicas de apertura y cerradura. Se utiliza un círculo como 
elemento estructural, con un radio de entre 8-10. Las operaciones morfológicas permiten eliminar el ruido, manteniendo intactos los niveles de intensidad de gris del termograma. Además, debido a que el tamaño del cuello es menor al del rostro y los hombros, la localización del rostro se realiza exitosamente.

\subsection{Eliminación de parte del fondo negro}

Luego de segmentar las imágenes térmicas, se observa que en la mayoría de ellas, el fondo negro ocupa un mayor espacio que el rostro mismo. Con el objetivo de que el descriptor FEN utilice la mayor cantidad de información térmica posible durante el procesos de reconocimiento, se procedió a eliminar una proporción del fondo negro mediante el recorte de la imagen alrededor del rostro. Este procedimiento reduce el tamaño de la imagen de $320 \times 240$ píxeles a $195 \times 230$ píxeles.

\subsection{Aplicación del modelo de perfusión sanguínea y/o del método de extracción de vasos sanguíneos}

El modelo de perfusión sanguínea se aplica en cada uno de los termogramas, posteriormente, el método de extracción de vasos sanguíneos se implementa en los termogramas. Es necesario hacer mención que solamente la primera metodología aplica el modelo de perfusión sanguínea en los termogramas. En el caso de la segunda metodología, se omite el modelo de perfusión sanguínea, y únicamente se hace uso del método de extracción de vasos sanguíneos.

\subsection{División del termograma y aplicación del descriptor FEN}

De acuerdo con lo descrito en la Sección 3.1, cada uno de los termogramas es dividido en 9 regiones dimensionales del mismo tamaño. Por cada una de las 9 sub-imágenes en que se han dividido cada una de las imágenes térmicas, se usa el descriptor FEN para obtener un vector de 9 dimensiones que representa la medida de la compacidad de las sub-imágenes que constituyen un rostro térmico.

\subsection{Clasificación por medio de una máquina de vector soporte y un bosque de aleatorio}

La máquina de vector soporte empleada para clasificar los termogramas, utiliza un kernel de función de base radial de tamaño 0.5 y un margen con dureza de 4.

Para el caso del bosque aleatorio, se emplean 950 árboles de decisión, la profundidad de los árboles se establece en 100. El número máximo de características usadas para definir la mejor partición, se define en 2, y finalmente, el número mínimo de datos permitidos en cada nodo terminal tiene un valor de 1 .

Tanto para la máquina de vector soporte como para el bosque aleatorio, se empleó una técnica de optimización de parámetros que utiliza la validación cruzada en una búsqueda en rejilla (grid search). 
Esta técnica se denomina grid search cross-validation y permite evaluar distinas combinaciones de los parámetros por medio del método de validación cruzada [20]. Luego de la evaluación, la técnica de grid search cross-validation determina el modelo óptimo para el clasificador en cuestión.

\section{Resultados}

Para realizar los experimentos propuestos en el presente artículo, se utilizó la base de datos Terravic IR. En la página web de descarga de esta base de datos, aparecen como disponibles las colecciones de imágenes correspondientes a 20 individuos, sin embargo, sólo es posible descargar las colecciones de 18 individuos. Tomando en cuenta lo anterior, el contenido de la base de datos es de 22,784 imágenes infrarrojas con dimensiones de $320 \times 240$, captadas en la longitud de onda del infrarrojo lejano y correspondientes a 18 individuos.

Los termogramas contenidos en esta base de datos, presentan variaciones en las posiciones del rostro (frente, izquierda, derecha) y en el entorno (interior, exterior), además de contener accesorios como lentes y gorras. En la Figura 3 se muestran algunas imágenes de esta base de datos.

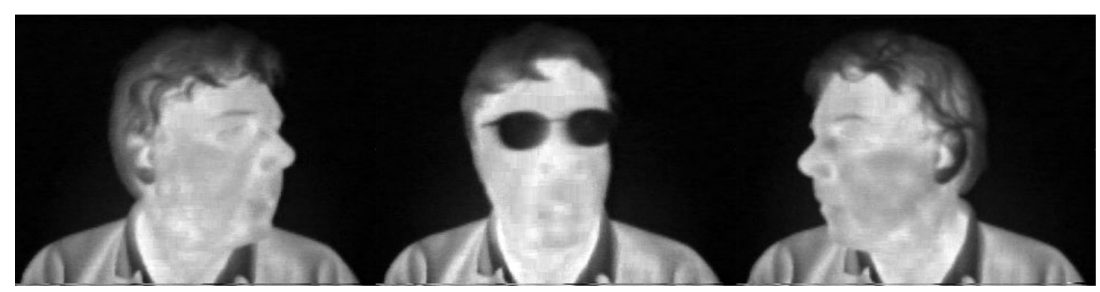

Fig. 3. Ejemplos de imágenes térmicas de la base de datos Terravic IR.

Para llevar a cabo la clasificación, tanto el conjunto de entrenamiento, como el conjunto de prueba, se conformaron con 100 imágenes térmicas aleatorias de cada uno de los individuos de la base de datos Terravic IR. Como parte de las dos metodologías para el reconocimiento de rostros que son propuestas en este artículo, se llevó a cabo la clasificación de las imágenes térmicas por medio de una máquina de vector soporte y un bosque aleatorio. En la Tabla 1 se muestran los resultados obtenidos cuando la clasificación se realiza de acuerdo con la primera metodología, mientras que la tasa de desempeño bajo el enfoque de la segunda metodología, se expone en la Tabla 2. La descripción de las metodologías se detalló en la Sección 4. 
Tabla 1. Tasa de desempeño para el sistema de reconocimiento de rostros propuesto en la metodología 1.

\begin{tabular}{cc}
\hline Algoritmo & Tasa de desempeño \\
\hline Máquina de vector soporte & $92 \%$ \\
Bosque aleatorio & $90.61 \%$ \\
\hline
\end{tabular}

Tabla 2. Tasa de desempeño para el sistema de reconocimiento de rostros propuesto en la metodología 2.

\begin{tabular}{cc}
\hline Algoritmo & Tasa de desempeño \\
\hline Máquina de vector soporte & $91.5 \%$ \\
Bosque aleatorio & $89.11 \%$ \\
\hline
\end{tabular}

Tabla 3. Matriz de confusión de la máquina de vector soporte de la metodología 1.

\begin{tabular}{|cccccccccccccccccc|}
\hline \multicolumn{10}{c}{} \\
\hline 0 & 0 & 0 & 0 & 0 & 0 & 0 & 0 & 0 & 0 & 0 & 0 & 0 & 0 & 0 & 0 & 0 & 0 \\
0 & 100 & 0 & 0 & 0 & 0 & 0 & 0 & 0 & 0 & 0 & 0 & 0 & 0 & 0 & 0 & 0 & 0 \\
0 & 0 & 90 & 0 & 6 & 1 & 0 & 0 & 0 & 1 & 0 & 0 & 0 & 0 & 0 & 0 & 2 & 0 \\
0 & 0 & 0 & 94 & 1 & 4 & 0 & 1 & 0 & 0 & 0 & 0 & 0 & 0 & 0 & 0 & 0 & 0 \\
0 & 0 & 3 & 2 & 86 & 4 & 0 & 0 & 0 & 2 & 0 & 1 & 1 & 0 & 0 & 1 & 0 & 0 \\
0 & 0 & 0 & 1 & 7 & 90 & 1 & 0 & 0 & 0 & 0 & 0 & 0 & 0 & 0 & 0 & 1 & 0 \\
1 & 0 & 0 & 0 & 0 & 0 & 98 & 0 & 0 & 1 & 0 & 0 & 0 & 0 & 0 & 0 & 0 & 0 \\
0 & 0 & 0 & 1 & 0 & 0 & 0 & 99 & 0 & 0 & 0 & 0 & 0 & 0 & 0 & 0 & 0 & 0 \\
0 & 1 & 0 & 0 & 0 & 0 & 0 & 0 & 98 & 0 & 0 & 0 & 0 & 0 & 0 & 1 & 0 & 0 \\
0 & 0 & 0 & 0 & 2 & 1 & 0 & 0 & 0 & 84 & 0 & 0 & 1 & 0 & 0 & 10 & 0 & 2 \\
0 & 1 & 0 & 0 & 0 & 0 & 0 & 0 & 0 & 0 & 94 & 1 & 1 & 1 & 2 & 0 & 0 & 0 \\
0 & 0 & 0 & 0 & 0 & 0 & 0 & 0 & 0 & 0 & 1 & 91 & 2 & 0 & 1 & 0 & 3 & 2 \\
0 & 0 & 0 & 0 & 0 & 0 & 0 & 0 & 0 & 1 & 3 & 0 & 95 & 0 & 0 & 0 & 0 & 1 \\
0 & 0 & 2 & 0 & 2 & 0 & 0 & 0 & 0 & 0 & 5 & 2 & 0 & 82 & 1 & 0 & 2 & 4 \\
0 & 0 & 0 & 0 & 0 & 0 & 0 & 0 & 0 & 1 & 2 & 0 & 0 & 1 & 90 & 2 & 3 & 1 \\
0 & 3 & 0 & 0 & 0 & 0 & 0 & 0 & 0 & 0 & 3 & 0 & 1 & 0 & 0 & 90 & 2 & 1 \\
0 & 0 & 1 & 0 & 0 & 1 & 0 & 0 & 0 & 4 & 0 & 1 & 2 & 0 & 0 & 6 & 80 & 5 \\
1 & 0 & 0 & 0 & 0 & 0 & 1 & 0 & 0 & 5 & 0 & 1 & 1 & 3 & 0 & 0 & 2 & 86
\end{tabular} \mid alidas esperadas


Los resultados obtenidos indican que con ambas metodologías se alcanza una alta precisión en el reconocimiento de rostros, y al comparar el rendimiento de los clasificadores, se observa que la máquina de vector soporte es superior al bosque aleatorio en las dos metodologías. Sin embargo, es importante destacar que las tasas de reconocimiento son muy similares en las dos metodologías, y por la tanto, se infiere que la combinación del modelo de perfusión sanguínea con el método de extracción de la red de vasos sanguíneos del rostro, no aporta una mejora considerable al enfoque propuesto por Buddharaju et al., y en cambio, provoca un aumento en el uso de recursos computacionales.

De acuerdo con los resultados anteriores y buscando ahondar más en el análisis de la clasificación de cada uno de los individuos, en la Tabla 3 se muestra la matriz de confusión creada a partir de la clasificación por medio de la máquina de vector soporte de la metodología 1.

\section{Conclusiones}

En este artículo, se realizó un análisis de los dos métodos fundamentales para la extracción de características invariantes del rostro térmico: la extracción de la red de vasos sanguíneos, propuesta por Buddharaju et al. y la extracción de la perfusión sanguínea, propuesta por Wu et al. Se expuso una metodología que implementa el método de Buddharaju et al. sobre el modelo de Wu et al. y al mismo tiempo, se presentó otra metodología en la que únicamente se aplica sobre los termogramas el método de extracción de la red de los vasos sanguíneos. Ambas propuestas muestran un comportamiento robusto ante los termogramas contenidos en la base de datos Terravic IR, que son clasificados por medio de una máquina de soporte vectorial y un bosque aleatorio, y que toman como datos de entrada a los termogramas divididos en nueve secciones, de acuerdo con el descriptor FEN. Los resultados de clasificación evidencian que el FEN efectivamente aprovecha la representación de los termogramas como redes de vasos sanguíneos; sin embargo, también se observa que el modelo de perfusión sanguínea no aumenta de forma considerable la tasa de reconocimiento cuando es usado con el método de extracción de la red de vasos sanguíneos. Para nuestro trabajo futuro, se buscará hacer uso de diferentes descriptores para comparar directamente el modelo de Wu et al. con el método de Buddharaju et al. Esto nos permitirá profundizar en las ventajas y desventajas que ofrecen dichos métodos.

Agradecimientos. Juan Humberto Sossa Azuela agradece al Instituto Politécnico Nacional y al CONACYT por el apoyo económico brindado para la realización de este proyecto, en el marco de los proyectos 20180730 y 65 (Fronteras de la Ciencia). Raúl Aguilar Figueroa agradece al CONACYT por la beca otorgada por el CONACUY para llevar a cabo sus estudios de maestría. Agradece también al Centro de Investigación en Computación del IPN por la oportunidad para realizar una estancia de investigación en el Laboratorio de Robótica y Mecatrónica. 
Estudio comparativo del reconocimiento de rostros térmicos basado en características invariantes

\section{Referencias}

1. Akhloufi, M., Bendada, A., Batsale, J.C.: State of the art in infrared face recognition. Quantitative InfraRed Thermography Journal 5(1), 3-26 (2008), http://www.tandfonline.com/doi/abs/10.3166/qirt.5.3-26

2. Breiman, L.: Random forests. Machine Learning 45(1), 5-32 (2001)

3. Buddharaju, P., Pavlidis, I., Tsiamyrtzis, P.: Pose-invariant physiological face recognition in the thermal infrared spectrum. In: Proceedings of the IEEE Computer Society Conference on Computer Vision and Pattern Recognition, vol. 2006 (2006)

4. Buddharaju, P., Pavlidis, I., Tsiamyrtzis, P.: Physiology-Based Face Recognition 1, 354-359 (2005)

5. Buddharaju, P., Pavlidis, I., Tsiamyrtzis, P., Bazakos, M.: Physiology-Based Face Recognition in the Thermal Infrared Spectrum. IEEE Transactions on Pattern Analysis and Machine Intelligence 29(4), 613-626 (2007)

6. Chollet, F.: Deep learning with Python. Manning (2017)

7. Cortes, C., Vapnik, V.: Support-Vector Networks. Machine Learning 20(3), 273-297 (1995)

8. Ghiass, R., Arandjelović, O., Bendada, A., Maldague, X.: Infrared face recognition: A comprehensive review of methodologies and databases. Pattern Recognition 47(9), 2807-2824 (2014)

9. Ghiass, R., Arandjelovi, O., Bendada, H., Maldague, X.: Vesselness Features and the Inverse Compositional AAM for Robust Face Recognition Using Thermal IR. pp. 357-364 (2013)

10. Hsu, C.W., Chang, C.C., Lin, C.J.: A practical guide to support vector classification 101, 1396-1400 (2003)

11. Kong, S., Heo, J., Abidi, B., Paik, J., Abidi, M.: Recent advances in visual and infrared face recognition - A review 97(1), 103-135 (2005)

12. Konuk, U.: Infrared face recognition. Master's thesis, The Graduate School of Natural and Applied Sciences of Middle East Technical University (2015)

13. Li, S., Chu, R., Liao, S., Zhang, L.: Illumination invariant face recognition using near-infrared images. IEEE Transactions on Pattern Analysis and Machine Intelligence 29(4), 627-639 (2007)

14. Li, S., Chu, R., Ao, M., Zhang, L., He, R.: Highly Accurate and Fast Face Recognition Using Near Infrared Images. pp. 151-158 (2006)

15. Li, S., His-Face-Team: AuthenMetric F1: A Highly Accurate and Fast Face Recognition System. ICCV2005 Demos, October 15 (2005)

16. Li, S., Zhang, L., Liao, S., Zhu, X., Chu, R., Ao, M., Ran, H.: A Near-infrared Image Based Face Recognition System. pp. 455-460 (2006), http://ieeexplore.ieee.org/lpdocs/epic03/wrapper.htm?arnumber=1613061

17. Prokoski, F.: History, Current Status, and Future of Infrared Identification (2000)

18. Prokoski, F., Riedel, R., Coffin, J.: Identification of Individuals by Means of Facial Thermography. In: International Carnahan Conference on Security Technology: Crime Countermeasures, pp. 120-125 (1992)

19. Santiago-Montero, R., Lopez-Morales, M.A., Sossa, J.H.: Digital shape compactness measure by means of perimeter ratios. Electronics Letters 50(3), 171-173 (January 2014)

20. VanderPlas, J.: Python Data Science Handbook. O'Reilly Media (2017)

21. Wu, S., Gu, Z., Kia, A., Sim, H.: Infrared facial recognition using modified blood perfusion. pp. 0-4 (2007) 


\section{Raúl Aguilar Figueroa, Raúl Santiago Montero, Juan Humberto Sossa Azuela}

22. Wu, S., Song, W., Jiang, L., Xie, S., Pan, F., Yau, W., Ranganath, S.: Infrared face recognition by using blood perfusion data. pp. 320-328 (2005)

23. Yoshitomi, Y., Miyaura, T., Tomita, S., Kimura, S.: Face Identification Using Thermal Image Processing. pp. 374-379 (1997)

24. Zhang, D., Lu, G.: Review of shape representation and description techniques. Pattern Recognition 37(1), 1-19 (2004) 